\title{
Peertechz
}

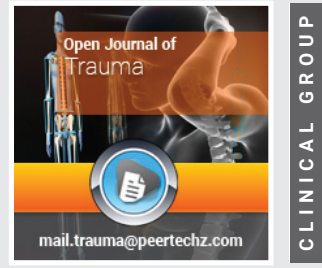

\section{Management of the pregnant trauma patient: A literature study}

\author{
Louise D' Argent, Sandra Verelst and Marc Sabbe*
}

Emergency Department, UZLeuven, Herestraat 49, 3000 Leuven, Belgium

Received: 06 August, 2020

Accepted: 17 August, 2020

Published: 18 August, 2020

*Corresponding author: Marc Sabbe, Emergency Department, UZLeuven, Herestraat 49, 3000 Leuven, Belgium, E-mail: marc.sabbe@uzleuven.be

Keywords: Pregnancy; Trauma; Management; Perimortem caesarean section and Perimortem caesarean delivery

https://www. peertechz.com

Check for updates

\section{Abstract}

Background: Trauma frequently occurs during pregnancy and can affect both mother and unborn child. It is essential to consider pregnancy in every female trauma patient of reproductive age. The management of a pregnant trauma patient is often considered to be complicated.

Main body: Therefore, it is important to follow a clear management protocol of the appropriate approach of the pregnant trauma patient. Furthermore, some pregnancy-specific complications of trauma such as placental abruption, uterine rupture, premature uterine contractions and their management are presented.

In pregnancy, the ABCDEF approach is used during the primary survey with the ' $F$ ' standing for fetus. Fetal vital signs are an important indicator of the maternal condition, and fetal outcome depends on maternal wellbeing. Concerning the secondary survey, a physical examination as well as indicated technical examinations should be performed with fetal monitoring being essential in a viable pregnancy. In case of non-reassuring findings, emergency caesarean section or laparotomy may be indicated.

In case of cardiac arrest, advanced trauma life support management should be started and the aetiology of cardiac arrest should be sought using the BEAUCHOPS tool. Early airway protection with adequate oxygenation and ventilation as well as effective chest compressions with tilting of the mother or manual displacement of the uterus, are essential.

Conclusion: A systematic approach (ABCDEF) of the traumatised pregnant patient is even more essential than in the non-pregnant patient. Saving the mother is crucial if you also want to save the unborn child.

\section{Abbreviations}

AFE: Amniotic Fluid Embolism; CPR: Cardiopulmonary Resuscitation; CTG: Cardiotocography; DIC: Diffuse Intravascular Coagulation; DPL: Diagnostic Peritoneal Lavage; FAST: Focused Assessment with Sonography for Trauma; FHR: Fetal Heart Rate; FMH: Fetomaternal Haemorrhage; GA: Gestational Age; IM: Intramuscular; ISS: Injury Severity Score; KB test: Kleihauer-Betke test; LMWH: Low Molecular Weight Heparines; NPV: Negative Predictive Value; MVA: Motor Vehicle Accident; PA: Placental Abruption; PCS: Perimortem Caesarean Section; ROM: Rupture of Membranes; UR: Uterine Rupture

\section{Background}

Trauma complicates approximately $7 \%$ of pregnancies and is thereby the leading cause of non-obstetric morbidity and mortality during pregnancy [1-10]. The risk of traumatic injury increases with the advancement of pregnancy $[4,8,10,11]$. Pregnancy as such does not increase morbidity or mortality following trauma but leads to different mechanisms and patterns of injury with an increased vulnerability to abdominal injury $[4,6,10,12]$. One needs to bear in mind that trauma affects both mother and unborn child, with the latter having a higher mortality rate $[4,13]$.

The spectrum of trauma severity is broad. Severe trauma often leads to fetal loss but minor trauma is responsible for the majority $(60-70 \%)$ of fetal deaths due to its higher prevalence $[2,4,10,12,14]$. However, the likelihood of adverse fetal outcome is often unpredictable and does not correlate with the degree of trauma $[4,15,16]$. 
The most common causes are Motor Vehicle Accidents (MVA), falls, assaults and burns with the order depending on the world region and the rate of intimate partner violence $[1,2,4-10,13]$. Blunt trauma is more prevalent than penetrating trauma $[5,7,9]$.

Fetal death following trauma is mostly caused by placental injury or maternal death $[1,4,6,8-10,14,17]$. Especially in the first trimester, when the uterus is less prone to direct injury, fetal death is often due to fetal hypoperfusion following maternal hypovolemia or hypotension [1]. Maternal death following MVA is mostly caused by head injury or intraabdominal haemorrhage $[1,2,8,10]$

ISS is a valuable predictor of maternal outcome but it does not seem to accurately predict fetal outcome. Although having a high specificity, several studies postulate that even a low ISS can be associated with adverse fetal outcomes $[2,13,14,17,18]$.

There are several reasons why the management of the pregnant trauma patient is considered difficult: anatomical and physiological changes; difficult identification of pregnancy specific complications; different mechanisms leading to potential injuries; rarity of presentation; hard risk stratification; difficult prediction of outcome and fear of radiation exposure. Furthermore, it concerns two patients resulting in a cognitive burden and an incorrect diversion of attention towards the fetus. The need for a multidisciplinary approach further jeopardizes an uncomplicated management $[4-7,9,10,17,18]$.

We provide an overview of the most important changes in pregnancy and their consequences as well as some important complications following trauma.

Different databases were searched including Pubmed, Cochrane, Medline and Embase. MeSH terms used were pregnancy, multiple trauma, wounds and injuries, and emergency treatment. Other terms used were polytrauma pregnant patient, trauma in pregnancy management, multiple trauma, pregnancy, trauma, management, perimortem caesarean section and perimortem caesarean delivery. A publication date filter was used including studies between 2008 and 2020. The obtained results were screened using title and/or abstract. The reference list of the selected articles was also browsed.

Exclusion criteria were: Not written in English, French or Dutch; no full-text available; case reports and series.

\section{Results}

A total of 30 articles were included in the qualitative synthesis (Figure 1).

\section{Anatomical and physiological changes}

The management of a pregnant trauma patient is complicated due to anatomical and physiological changes of pregnancy [12]. Specifically, these changes can mask and mimic symptoms of injury but also predispose to trauma and alter the pattern of injury [10]. Therefore, understanding these modifications facilitates evaluation and management $[2,4,10]$. Table 1 gives an overview of the specific changes and their consequences.

\section{Pregnancy associated complications}

In case of suspected or confirmed obstetrical complications, an urgent obstetric consultation is always warranted [6,17].

\section{Placental abruption}

Placental abruption (PA) is a major obstetrical complication following trauma, especially MVA $[7,12,26]$. The prevalence varies widely but can reach up to $50 \%[6-10,12,14,15]$. It should also be considered following minor trauma, especially later in pregnancy $[6,20]$. It is the most common cause of fetal death with maternal survival following blunt trauma [6,8-10,13,1518]. Fetal mortality rate following PA can reach up to $75 \%$ and increased risk of stillbirth may be due to undetected or delayed PA $[15,20]$. Preterm labour, urgent caesarean delivery, diffuse intravascular coagulation (DIC), haemorrhage and isoimmunisation are possible consequences of PA besides fetal death $[1,4,6,7,10,13,15,17,20]$.

PA is primarily a clinical diagnosis, based on suspicion $[6,17,20,26]$. Signs may be abdominal pain, vaginal bleeding or leakage of amniotic fluid, back pain, uterine tenderness with regular uterine contractions and contraction severity being out of proportion to cervical dilatation, a large uterus, or maternal hypovolemia $[2,4,6,7,9-11,15,17-20]$. The amount of vaginal bleeding does not indicate the severity of PA as the uterine cavity can accommodate the entire blood volume $[15,20]$. However, detecting PA may be challenging because signs are often absent, especially following minor trauma $[11,19,20,26]$.

Fetal monitoring using cardiotocography (CTG) is the most available technique in detecting PA with a negative predictive value (NPV) of $100 \%[4,6,10,12,13,16,21]$. Fetal distress is predictive of PA in $60 \%$ of all cases [19]. Fetal tracing may be reassuring until $30 \%$ of the placenta is separated from the uterus, whereas an uteroplacental separation of more than $50 \%$ is consistently fatal for the fetus $[6,15,19]$.

Excessive uterine activity with more than four contractions per hour may be indicative of PA. More than eight contractions per hour in the first four hours is consistent with the diagnosis of PA $[10,18,21]$. Eighty percent occur within six hours of monitoring but may be delayed for up to 48 hours or even longer $[1,2,5,6,13,15,20,21,27]$. However, PA has not been reported when there is less than 1 contraction every 10 minutes over a four hour period $[12,13,27]$.

Ultrasound may also help diagnosing PA since its positive predictive value is high, although its NPV is low $[1,2,4,6,7,10,11,14,15,17-21,26]$. It may show a retro- or preplacental haematoma, increased placental thickness, or a subchorionic or marginal collection. [1,6] Abdominal computed tomography may be more helpful, carrying a $96 \%$ overall accuracy rate $[17,22,26]$.

The management depends on the gestational age (GA) as

Citation: Argent LD, Verelst S, Sabbe M (2020) Management of the pregnant trauma patient: A literature study. Open J Trauma 4(1): 038-046. 


\section{PRISMA 2009 Flow Diagram}
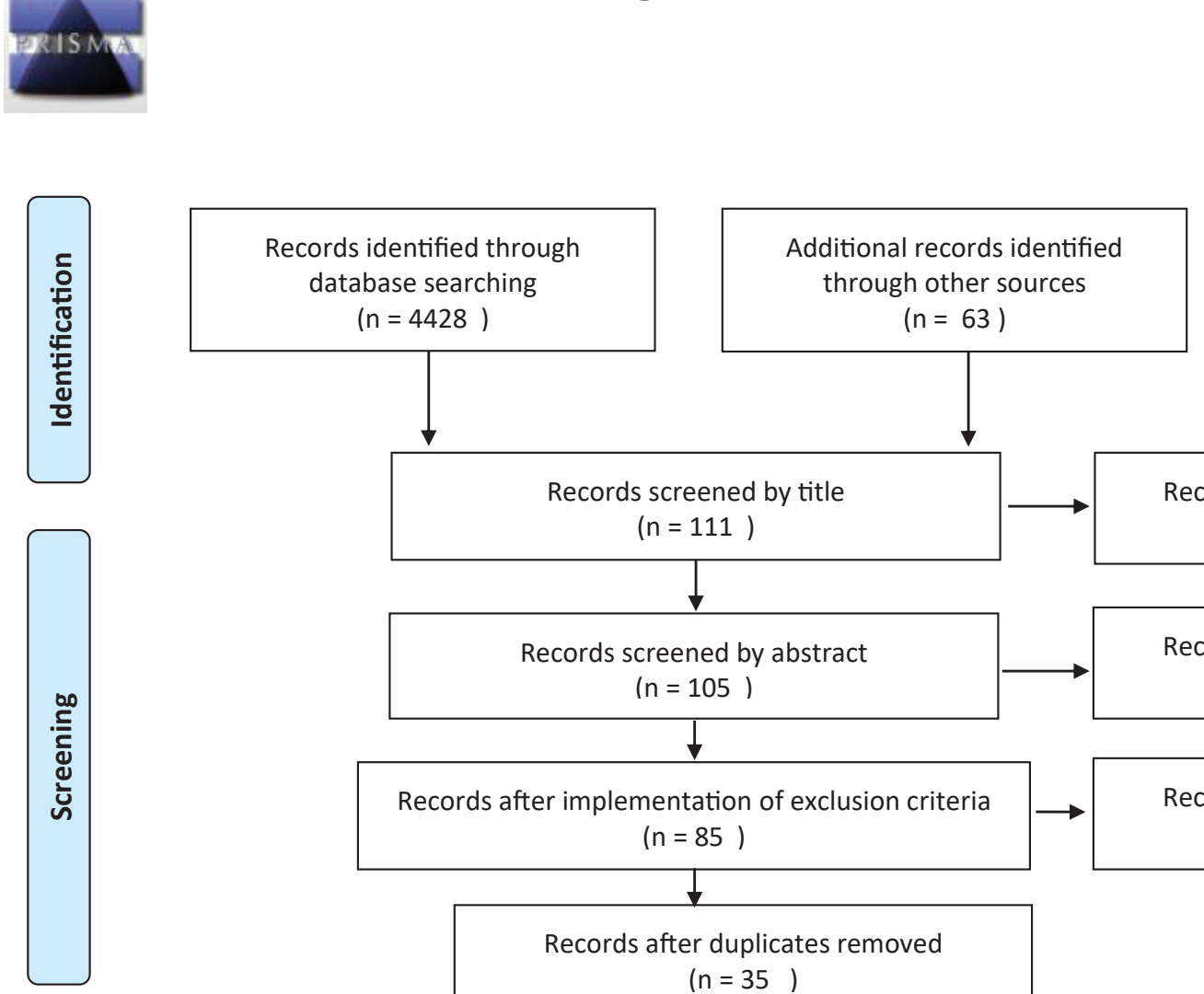

$(n=4428)$
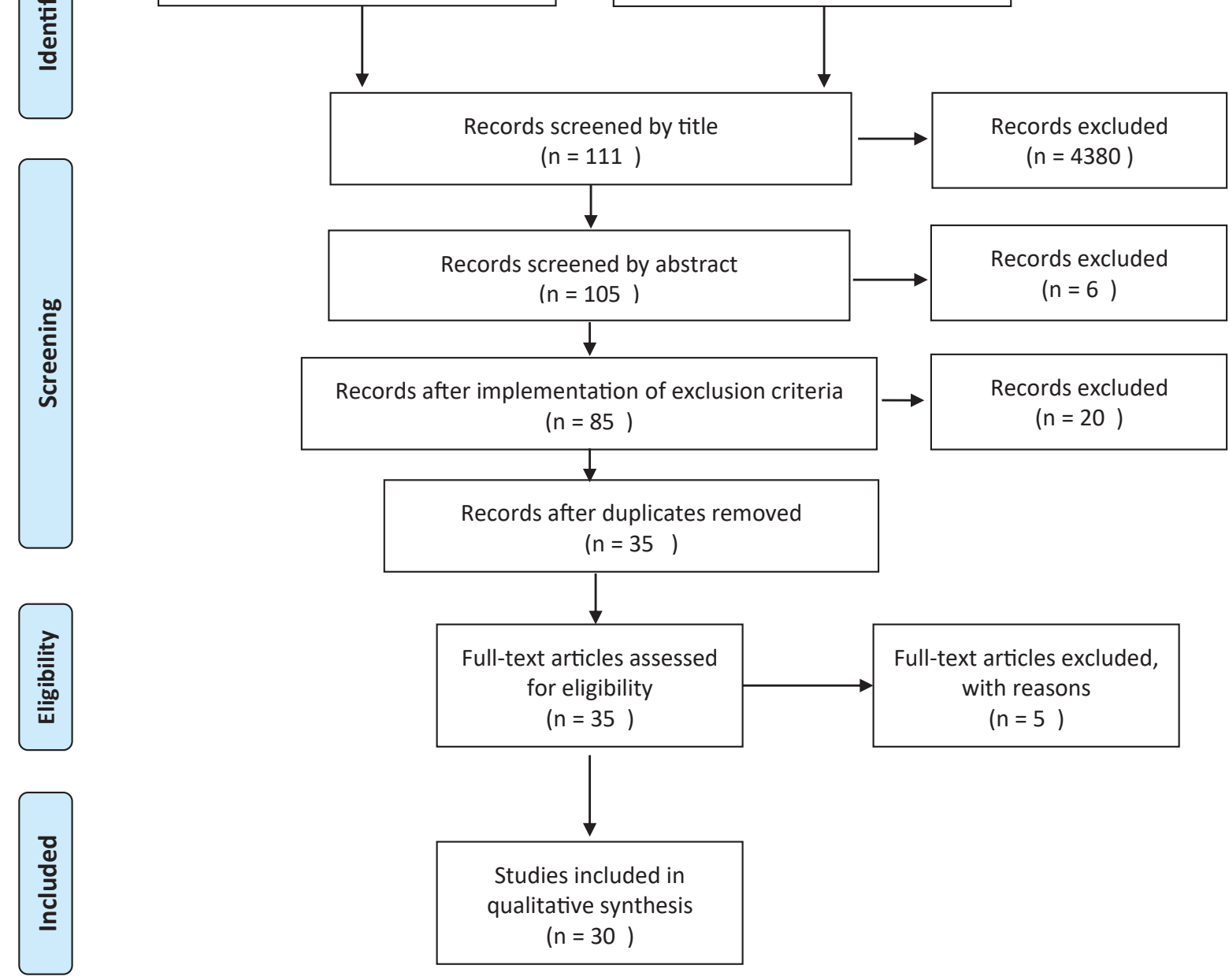

From: Moher D, Liberati A, Tetzlaff J, Altman DG, The PRISMA Group (2009). Preferred Reporting /tems for Systematic Reviews and MetaAnalyses: The PRISMA Statement. PLoS Med 6(7): e1000097. doi:10.1371/journal.pmed1000097

For more information, visit www.prisma-statement.org.

Figure 1: PRISMA 2009 flow diagram

well as on the maternal and fetal wellbeing. In case of preterm labour around the expected delivery date, vaginal delivery may be reasonable if both are stable [6]. In case of fetal compromise while the mother is stable and a GA is higher than 23-24 weeks, emergency caesarean section is recommended to prevent coagulopathy and its adverse consequences $[6,18,19,26]$. There is no consensus whether emergency caesarean section should be performed in case of maternal instability $[6,26]$. When PA seems mild, betamethasone administration may be considered. Furthermore, correction of haematological abnormalities and ultrasound follow up for evaluation of amniotic fluid and fetal growth are recommended. $[18,20,26]$.

\section{Premature uterine contractions}

The most common obstetric complication following trauma is premature contractions, especially if accompanied 
Table 1: Overview of the anatomical and physiological changes of pregnancy and their consequences.

\section{Changes of pregnancy}

Consequences

\section{Cardiovascular changes}

Mild hypotension and tachycardia $[1,2,4,6-10,14,17,19,20]$

Vital signs poor marker of maternal and uteroplacental circulation $[1,2,6-8,13,14,17,19-22]$

Peripheral vasodilation $[4,8,10]$

Hypervolemic state with increased cardiac output $[1,2,4-10,13,14,17,19-23]$

Warm, dry and pink skin, even following significant blood loss

Protective against haemorrhage and difficult detection of hypovolemia $[1,4-6,9,10,17,19,20]$ Changes in vital signs only apparent when 1,5-2 I acute blood loss

Physiological anaemia with lower oncotic pressure and arterial oxygen [4,5,7,8,1 More risk of pulmonary oedema [20]

$0,13,14,17,19,20,22,23]$

Hypercoagulable state $[4,8-10,14,17,19,20,22]$

Uterine blood flow depends on maternal mean art pressure $[4,6,17]$

Uterine/ovarian vessels engorged and sensitive to catecholamines

Inelastic and vascular placenta [2,4,6,8-10,13-15,17,19,20,23]

Pelvic blood flow increases and pelvic vessels are hypertrophied $[2,4,10,13,17$

Spleen engorgement $[4,8,10]$

Pituitary gland anterior lobe enlargement [19]

More dependent on cardiac output for oxygen delivery [20]

Predisposition to consumptive coagulopathy following bleeding $[4,17,22]$

Improved haemostasis but higher thromboembolism risk [4,8-10,14,19]

Hypovolemic shock following vessel injury, uterine rupture, PA [2,4,10,13,17,19-22]

Hypovolemia causes vasoconstriction of uterine vessels leading to fetal distress before occurrence of hypovolemic shock $[6,10,13,20]$

Brachial arterial pressure not similar to uterine arterial pressure [9]

Retroperitoneal bleeding when pelvic trauma or fracture $[4,8,10,13,15,17,19,20]$

More prone to rupture: common cause of abdominal haemorrhage $[4,8,10,17]$

More vulnerable to ischaemia/necrosis when traumatic shock [19]

\section{Gastro-intestinal changes}

Oesophageal sphincter relaxation and partial displacement into thorax

Delayed gastric emptying and decreased bowel motility

More gastric secretion with lower $\mathrm{pH}$

Compression of upper tract in supine position $[2,4,5,6,8,10,13,14,17,19,21,22]$

Superior displacement of intestines $[2,4,5,8,10,13,17,19,22]$

Uterus shields abdominal structures $[6,8,10]$

Abdominal desensitization due to stretching $[4,10,13,17]$

Intraabdominal organ displacement [4,5]

\section{Respiratory changes}

Vascular engorgement of respiratory tract causing oedema

$[2,4,6,8,10,14,17,18,21]$

Weight gain $[4,6,8,10,18]$ and Tongue enlargement $[4,8,10]$

Increased oxygen consumption $6,8,20$

Decreased functional residual capacity (FRC) 6,8 and compliance 6

Increased airway resistance 6

Raised diaphragm causing FRC decrease and increased minute ventilation (MV)

$[4,5,6,10,14,17,19,21,22]$

Increased tidal volume causing increased MV $[4,9,10,14,17,18,19,20,21,22,23]$

Increased MV leading to physiological hyperventilation with respiratory alkalosi and metabolic compensation: low $\mathrm{paCO}_{2}$, low $\mathrm{HCO}_{3}[4,6,10]$

FRC decreased and increased oxygen demand $[4,5,6,8,10,14,17,18,19,20,21,23$

24,25]

Airway closure when supine [19]

Reduced oxygen carrying capacity [23]

Urogenital changes

Compression of ureters and bladder by uterus: dilated ureters $[17,19]$

Superior displacement of bladder $[4,10,15,17,19]$

\section{Renal changes}

Increase in renal blood flow Glomerular filtration rate (GFR) increase with decrease of creatinine and urea nitrogen $[4,8,17,19,20]$

\section{Anatomical changes}

More than 20-24 weeks pregnancy: uterine compression of inferior vena cava in supine position $[1,2,4,5,6,8,9,10,11,14,17,18,20,21,23]$

Enlarged uterus with thinning wall $[4,7,10,13,22]$

Fetus becomes most anterior structure in abdomen [22]

Increased sacroiliac joint and symphysis laxity with widening of the joints

Weight gain and increased lordosis $[4,5,6,10,15,17,19,22]$

Rotation of the heart around its long axis, towards the left and superior [9]

Decreased anaesthetic requirements [19]

Higher aspiration risk $[2,4,5,6,8,9,10,13,14,15,17,18,19,22]$

Pregnancy complaints (nausea, vomiting and abdominal pain) can mimic symptoms following trauma [10]

Upper abdomen penetrating injury likely to injure bowels $[2,6,13,15,17,19]$

Lower abdominal penetrating injury likely to damage uterus and fetus $[5,6,10]$

difficult diagnosis of peritonitis or hemoperitoneum because guarding and rebound

tenderness less apparent and atypical pain referral $[4,6,10,13,17]$

Difficult airway $[2,4,6,8,10,17,18]$

Gastric tube insertion more difficult

Cardiopulmonary resuscitation (CPR) more difficult [21]

Bleeding possible when airway manipulation or gastric tube insertion $4,8,10$

Caution during thoracostomy and thoracentesis $[4,5,9,17,19]$

Penetrating chest trauma can be intra-abdominal [17]

Chest enlargement but FRC still lower [19,20,22]

Difficult ventilation [6,21] and less compensation in case of acidosis [17]

Concern for lower threshold for respiratory insufficiency $[4,10,20]$

Changes in maternal $\mathrm{pH}$ can influence fetal oxygen delivery [9]

Hypoxemia when apnoea or hypoventilation $[4,5,8,9,10,14,17,18,19,23,24,25]$

$\mathrm{PaO} 2<60 \mathrm{~mm} \mathrm{Hg}$ : immediate compromise of fetal oxygenation $[6,10,13]$

Rapid metabolic acidosis in case of hypoperfusion or hypoxia [8]

More susceptible to genitourinary injury and impaired urine outflow [15] Hydronephrosis and hydroureter $[17,19]$

Lower abdomen penetrating injury may injure bladder $[4,10,15,17,19]$

Pollakisuria: urine output poor indicator of shock

Renal dysfunction with normal creatinine and urea nitrogen values possible

Lower oncotic pressure with higher risk of pulmonary oedema [19]

Decreased preload and cardiac output (= Supine hypotensive syndrome), especially when hypovolemic $[2,4,5,6,9,10,14,18,19,20,22]$

Increase in venous pressure below uterus with venous pooling: exacerbation of pelvic placental or lower extremity bleeding and predisposing to DVT $[4,9,10]$

Further compromise of cardiac output during CPR [1,6,24,25]

Increased risk of direct trauma to uterus, fetus and placenta possibly leading to uterine

rupture, placental abruption and fetal injury $[4,7,10,13,14,19,20]$

Gait instability with predisposal to falls $[4,5,6,8,10,12,19,22]$

Pelvic fractures most common injury following blunt trauma $[4,10,15]$

Altered heart sounds with systolic murmur in $95 \%$ of patients [20]

Mild cardiomegaly, widened mediastinum [10,20]

ECG changes: left axis deviation, inverted or flattened T waves in III,V1,V2 and Q waves in III and aVF $[4,10]$

Need of adjustment of sedative doses [19]

Citation: Argent LD, Verelst S, Sabbe M (2020) Management of the pregnant trauma patient: A literature study. Open J Trauma 4(1): 038-046.

DOI: https://dx.doi.org/10.17352/ojt.000031 
by cervical changes $[4,10,18,19]$. Possible signs are regular painful contractions and vaginal bleeding, and therefore sometimes difficult to differentiate from PA $[9,10,17,18]$. The majority resolve spontaneously and those persisting are often pathologic in origin and hence, in itself, a contraindication for tocolytics [19].

\section{Rupture of membranes}

Following trauma, rupture of membranes (ROM) is possible causing preterm labour, umbilical cord prolapse with compression of umbilical vessels, infection or, if occurring in the second trimester, pulmonary hypoplasia or orthopaedic deformities $[6,9,13,19]$. If ROM is suspected, an external pelvic examination can assess vaginal fluid leakage and nitrazine paper can be used to distinguish amniotic fluid from normal vaginal secretions $[6,11,17]$. A digital vaginal examination should be avoided in order to reduce infection risk $[15,17,18]$.

\section{Preterm labour}

Preterm labour can follow PA, ROM or premature uterine contractions [6]. It is associated with a higher risk of preterm delivery and should therefore be evaluated in every pregnant trauma patient $[10,17,19]$. The risk of preterm delivery is two times higher in pregnant trauma patients [6]. In case of regular uterine contractions, a fibronectin test or cervical length assessment should be conducted in order to determine the risk of preterm labour [6].

\section{Uterine rupture}

A rare but life-threatening complication following trauma is an Uterine Rupture (UR) $[6,7,10,20,27]$. It mostly occurs after a direct, high energy, blunt or penetrating injury to the abdomen $[4,6,11,13,17]$. It should be suspected in assault victims and patients with a pelvic fracture, direct perineal trauma or penetrating injury to the pelvis $[4,10,19]$.

UR mostly occurs later in pregnancy and in women with an uterine scar $[4,6,10,13]$. In an unscarred uterus, the fundus or the posterior part is often involved. The extent of rupture varies widely up to complete uterine avulsion. An associated bladder injury is common $[4,6,10,20,27]$. UR encompasses a bad prognosis for both, reaching a fetal mortality rate of $100 \%$, while maternal mortality rate approaches $10 \%$ $[1,4,7,8,10,13,20,27]$.

Clinical signs may mimic those of PA, but may also be absent $[1,10,17]$. Physical examination is not reliable. It may reveal uterine tenderness, peritonitis, a bulging perineum or irregular uterine contours due to extrauterine fetal parts and a discrepancy between uterine size and GA $[6,9,10,17,19]$. Vital signs may be stable or indicate shock. Focused Assessment with Sonography for Trauma (FAST) may demonstrate abdominal free fluid. Fetal monitoring may demonstrate distress and ultrasound can be diagnostic but a definitive diagnosis can only be made by laparotomy $[6,14,17,27]$. Therefore urgent exploratory surgery may be recommended following abdominal trauma to reveal the extent of damage to uterus and adjacent organs as well as to avoid haemorrhage $[1,6,10,19]$. If the uterus is severely damaged, hysterectomy is recommended to prevent further deterioration of the maternal condition $[1,19]$.

\section{Fetomaternal haemorrhage}

Fetomaternal haemorrhage (FMH) represents a transplacental passage of fetal blood into the maternal circulation [19]. It is only a concern from 12 weeks of gestation on, when the uterus becomes an intra-abdominal organ [19]. FMH occurs in $10-30 \%$ of pregnant trauma patients $[1,2,4,6,10,13,15,17-20]$. It is associated with an anteriorly placed placenta and PA $[1,4,10,13]$. Signs may be uterine tenderness, contractions and vaginal bleeding but mostly, FMH occurs sub-clinically $[4,6,10,11,13,17]$. Extensive FMH is only observed in major trauma and is usually clinically evident with fetal anaemia, hypoxia, distress or death from exsanguination $[1,4,6,10,11,13,19,20]$. Haemolytic disease of the newborn and neonatal neurologic damage are also possible $[1,11,13,19]$. For the rhesus negative mother carrying a rhesus positive fetus, isoimmunisation is a risk $[4,10,11,13,15]$. The Kleihauer-Betke (KB) test quantifies the presence of fetal cells in the maternal circulation. However, its use is subject of discussion $[1,2,4,10,11,15,17,18,21,26]$. It has a low sensitivity but may be useful in quantifying FMH in the group susceptible for isoimmunisation. $[4,12,20]$ There is no consensus if it could be useful in rhesus positive pregnant women to determine the degree of FMH $[6,10,11,16,19,21,22,26]$. Fetal monitoring is more reliable $[19,20,22]$. Fetal middle cerebral arterial doppler showing elevated peak systolic velocity is correlated with fetal anaemia and thus FMH $[2,19]$. In some cases, an emergency caesarean section is indicated. $[6,19]$ There may also be a limited role for intrauterine transfusions [26].

\section{Amniotic fluid embolism}

Amniotic Fluid Embolism (AFE) is a rare complication of trauma [18]. It may occur following severe trauma due to a sudden increase in amniotic fluid pressure at impact and the presence of vascular wounds [14]. Its incidence and mortality rate varies but it is certainly associated with a high maternal mortality rate $[9,18,19,22]$. Signs may include respiratory distress with hypoxia, shock or severe hypotension, seizures, coma and cardiac arrest $[15,18,22]$. Up to $50 \%$ will develop DIC or other coagulation disorders $[9,14,18,22]$. A high index of suspicion is warranted [18]. Echocardiography may show right heart strain or failure and increased pulmonary artery pressure [22]. Management includes supportive care and cardiopulmonary bypass should be considered $[15,22]$.

\section{Non-pregnancy associated complications: Pelvic fracture}

Pelvic fractures are one of the most common non-obstetric complications of blunt trauma sustained in pregnancy, possibly due to increased pelvic laxity [15]. Pelvic fracture is an independent risk factor for poor fetal outcome and together with PA, it is the most common cause of fetal death following MVA $[5,10,14,15,19]$.

Citation: Argent LD, Verelst S, Sabbe M (2020) Management of the pregnant trauma patient: A literature study. Open J Trauma 4(1): 038-046. 
Due to the vascular changes, retro- and intraperitoneal haemorrhage can lead to fetal compromise and even maternal death $[2,4,9,10,13,15,17,19,27]$. When the fetal head is engaged, fetal brain haemorrhage and skull fracture are common $[1,4,6,10,13,15,20]$. Pelvic fractures also increase the risk of injuries to other organs such as bowel, uterus, bladder and urethra $[2,4,9,10,13,15,19,27]$.

Diagnosis is based on physical examination supplemented by pelvic $X$ ray. $[10,27]$ A vaginal speculum examination should be performed when a pelvic fracture is suspected, in order to exclude bleeding, ROM, vaginal lacerations or bony fragments $[1,11]$.

Management may resemble that of the nonpregnant patient [26]. Pelvic binders are recommended for open book pelvic fractures to control haemorrhage [17]. Angioembolisation is a useful minimally invasive procedure to control haemorrhage from pelvic vessels not involved in the uterine circulation, although containing a high radiation dose $[5,15]$.

\section{General management}

Pregnancy should be suspected in every female trauma patient of childbearing age until proven otherwise $[4,6,17]$. Thus, urine and serum pregnancy tests should be conducted in this female age group $[4,6,19]$.

The management resembles that of a non-pregnant trauma patient $[10,12,14,15,17,22]$. However, some important considerations should be made regarding primary and secondary survey, as well as the management of cardiac arrest.

\section{Primary survey}

In pregnant women, the ABCDEF algorithm should be followed where $\mathrm{F}$ stands for fetus $[2,20]$. This indicates that the fetus is enclosed in the primary survey but after the initial assessment and stabilisation of the mother $[4,6,8-10,19,26]$. Fetal vital signs can be an important predictor of the maternal condition and can therefore guide maternal management [4$7,10,17,18,20]$. On the other hand, fetal wellbeing depends on maternal wellbeing and most interventions to help the mother will simultaneously benefit the fetus. $[2,8,12-14,16,18,20]$ Interventions for fetal benefit should only be performed after maternal stabilisation $[2,6,9,26]$. Re-evaluation of the patient is essential to immediately detect subtle deteriorations [16].

Care should be taken while performing a left lateral tilt, preferably with a secured spine $[4,6,8,10]$. The threshold for intubation is lower in pregnant patients [17]. Preoxygenation with bag valve mask ventilation increases the risk of aspiration and therefore preoxygenation should be achieved by other techniques [11,17]. The $\mathrm{pCO}_{2}$ goal after intubation is 28 $35 \mathrm{mmHg}$ to prevent respiratory alkalosis and decrease of uteroplacental flow [17].

The threshold for volume resuscitation is also lower in pregnancy [19]. However, an exaggerated fluid resuscitation can lead to pulmonary oedema, and thus balanced fluid resuscitation is crucial [17]. Since vasopressors compromise uteroplacental perfusion, they should only be administered in case of hypotension that is fluid unresponsive $[4-6,10]$. Administration of bicarbonate should be used with caution [6].

The sensitivity and specificity of FAST is comparable with non-pregnant trauma patients, however, differentiation between intra- and extrauterine fluid can be challenging $[4,9]$.

Traumatic brain injury is a major cause of maternal morbidity and mortality [15]. Also intracranial haemorrhage due to gestational diabetes mellitus and pregnancy induced hypertension should be considered [9].

\section{Secondary survey}

The secondary survey further evaluates the fetal state and the presence of uterine injury $[7,8,20]$. An obstetric consult is needed, especially when the fetus is potentially viable obstetrical complications are suspected, or delivery is being considered $[6,14,17]$.

GA must be estimated to assess viability, to direct management and to estimate radiation effects of radiologic examinations $[4,11,14,18]$. Comorbidities and complications of the current or previous pregnancies and deliveries should be assessed since they can affect management $[4,6,17,19]$. Inquiries should be made about fetal movement and specific symptoms such as contractions, vaginal leakage of fluid or blood, haematuria, or pain $[4-6,12,17]$.

During physical examination, the entire body is inspected and palpated, including the screening for signs of physical abuse $[1,11]$. In order to assess complications, an abdominal and pelvic examination must be conducted [15]. In the absence of concerning signs and symptoms, an external pelvic examination may be sufficient [17]. However, in case of any concern, a sterile speculum examination should be performed to detect bleeding and identify its aetiology; assess cervical dilation and effacement; detect a prolapsed cord, a bulging perineum, a haematoma, vaginal lacerations, bony fragments, or ROM; assess the fetal position; or to identify fetal parts $[1,4,5,6,9-12,15,17,18,22]$. A rectal examination allows for the identification of blood and haematomas $[4,10]$. An ultrasound should be performed before the speculum examination to detect a placenta previa $[4,6,10,15,17,18]$. In case of a placenta previa, a digital vaginal examination should be avoided $[9,10,15,17,18]$.

An abdominal examination is essential in order to detect ecchymoses which can indicate visceral injury. Abnormal abdominal distention can be a sign of intra-abdominal bleeding or organ perforation. Abdominal tenderness and contractions should be assessed in addition to palpation of the uterus and assessment of the fundal height, which can help determine the GA or indicate an abruption when increased $[4,6,10,13,15,17,19]$. Unexplained hypovolemia and rib or pelvic fractures raise the suspicion for intra-abdominal trauma [9]. After 20 weeks GA, fetal heart sounds should be auscultated [10].

A biochemical examination is mostly performed in case of minor trauma, although it is not predictive for fetal outcome

Citation: Argent LD, Verelst S, Sabbe M (2020) Management of the pregnant trauma patient: A literature study. Open J Trauma 4(1): 038-046. 
$[3,12,17]$. In case of major trauma or when PA or internal bleeding is suspected or confirmed, an additional coagulation profile is warranted $[1,2,4,6,9,13,17,26]$. Since fibrinogen levels are increased during pregnancy, low to normal levels indicate consumptive coagulopathy, especially when combined with low platelets and high fibrin degradation products $[4,6,10,20,22]$. Severe coagulopathy may be indicative of PA, $\mathrm{AFE}$, or major bleeding [14]. An arterial blood gas is also of importance since maternal $\mathrm{pH}$ influences fetal oxygen delivery $[4,9,10]$. Additionally, the KB test may be helpful after 20 weeks of gestation in rhesus negative mothers when detecting more than 30 millilitres $(\mathrm{ml})$ of fetal blood thereby guiding rhesus immunoglobulin administration $[1,2,6,9,11,17,26,19]$. In case of major trauma with large volume $\mathrm{FMH}$, the $\mathrm{KB}$ test in combination with other features such as abdominal trauma and third trimester pregnancy can be a predictive risk factor for preterm labour and other adverse outcomes $[1,4,9,11,12,15,20,22]$. In case of minor trauma however, extended evaluation with fibrinogen levels, $\mathrm{KB}$ test and coagulation profiles should not be performed as they are not predictive of adverse outcomes [3,9].

Similar to the nonpregnant patient, radiologic examinations should be conducted as indicated [2,4,5,6,8$10,12,14,16,17,20,19,22]$. Fifty milligrays is considered the threshold at which there is an increased risk for the occurrence of fetal malformation, but this radiation dose is not reached with common radiologic examinations $[1,4,10,18,20,19,22,28]$. Preventive measures such as shielding of the uterus in case of non-abdominal studies, and low exposure techniques should be taken $[4,10,15,18,19]$. Contrast agents may be used, especially when non-ionated $[2,6,14,17,18]$. The benefit of timely diagnosing maternal injuries outweighs the possible radiation risk $[9,10,12,13,15,17,19,28]$.

Monitoring may improve outcome due to its high sensitivity for early identification of premature labour, PA and fetal distress, although the specificity is low $[2,6,7,10,12,15,17-$ 20]. Monitoring is less helpful in unstable patients [12]. Continuous CTG is indicated to rapidly detect changes $[2,5,6,8$ $13,15,16,17,19-21,26]$. Fetal heart rate (FHR) monitoring is a good indicator of fetal wellbeing, and acts as a supplemental maternal vital sign $[2,6,11,17,20]$. Fetal bradycardia and tachycardia are non-reassuring, with bradycardia being more threatening since it may indicate fetal hypoxia $[4,7,9,10,26]$. Fetal and maternal heart rate should be compared in order not to misidentify maternal tachycardia as the fetal pulse $[11,13]$. Additionally, a waveform interpretation is paramount with repetitive late decelerations and loss of variability with sinusoidal pattern indicating fetal asphyxia or anemia $[4,7,9,10,13,15,17,26]$. Contraction frequency and regularity are also monitored with CTG $[4,10,17]$. Uterine activity of more than four contractions per hour may be indicative of PA or preterm contractions $[7,18,21]$. Besides frequency, the intensity of contractions are predictive of complications [6]. There is no agreement concerning the duration of monitoring but at least four to six hours is recommended $[1,4-7,9,11-18,21,26]$. In the absence of abdominal pain or vaginal bleeding, combined with a reassuring fetal heart tracing, there is no risk when there is less than one contraction every 10 minutes over a four hour period. In that case, the patient may be discharged if laboratory evaluation is normal and maternal state is reassuring [1,2,4$7,10,12,13,17,20,27]$. An obstetrical ultrasound should be carried out prior to discharge [6]. The combination of fetal monitoring with ultrasound provides the highest sensitivity and specificity [19]. Fetal ultrasound should be performed urgently when delivery is being considered and GA is undetermined [6].

Since the risk for pulmonary embolism is increased during pregnancy in combination with immobilisation following trauma, the threshold for prophylactic heparin or Low Molecular Weight Heparines (LMWH) is lower $[4,9,10,15,20,26]$. Tranexamic acid, although not studied, can be administered in case of significant haemorrhage and is safe for the fetus $[9,14,17,26]$. Tocolytics to suppress preterm labour in patients with a viable fetus and cervical changes can be considered $[8,17,19,20]$. Different options exist including the Intramuscular (IM) administration of $12 \mathrm{mg}$ of betamethasone, although this may mask signs of shock due to its betamimetic effects $[1,17,21]$. Tocolytics should not be administered in case of fetal distress, maternal haemodynamic instability or if labour is considered to have a pathogenic etiology $[17,19]$. If labour and cervical changes progress despite tocolytics, the possibility of PA must be considered [1].

Three hundred micrograms $(\mu \mathrm{g})$ of rhesus immunoglobulins should be administered IM in rhesus negative pregnant women within 72 hours following trauma, irrespective of the KB test $[1,4,6,10-13,15-22,26]$. This dose covers up to $30 \mathrm{ml}$ of $\mathrm{FMH}$ $[6,13]$. During the first trimester, $50 \mu \mathrm{g}$ of immunoglobulin is sufficient [11]. An additional $300 \mu \mathrm{g}$ should be administered for every 15 to $30 \mathrm{ml}$ of $\mathrm{FMH}$ exceeding the initial $30 \mathrm{ml}$, determined by the $\mathrm{KB}$ test $[2,6,10,19]$. In case of major $\mathrm{FMH}$, an antibody screening should be conducted two to three days after the immunoglobulin administration [4].

In case of non-reassuring fetal heart tracings, maternal resuscitation is essential $[17,18]$. If there is further fetal deterioration, emergency caesarean section is recommended even if a PA is not clinically evident $[1,8,10,17,18]$.

Diagnostic peritoneal lavage (DPL) has largely been replaced by FAST although when FAST is ambiguous, DPL can be performed $[4,10,15,26]$. Abdominal CT may also be an alternative as it is also able to identify retroperitoneal damage [6]. The haemodynamically unstable pregnant trauma patient with a positive FAST or with stabbing or gunshot wounds to the abdomen should undergo an exploratory laparotomy $[4,9,10,17]$. This also applies the presence of meconium or vernix in the urine which suggest vesico-uterine rupture [15].

\section{Cardiac arrest}

Maternal cardiac arrest is rare, occurring in 1/30.000 pregnancies $[4,9,10,23,24,29]$. Survival is lower in the pregnant population [1]. Basic resuscitative efforts must be initiated and a resuscitation team activated immediately [4,29]. Due to diaphragmatic elevation and heart displacement in pregnancy, hand positioning should be slightly higher on the sternum, though there is no consensus $[4,10,15,21,26,29]$. Due to a

Citation: Argent LD, Verelst S, Sabbe M (2020) Management of the pregnant trauma patient: A literature study. Open J Trauma 4(1): 038-046. 
decreased chest wall compliance, breast hypertrophy and diaphragmatic elevation compression force should be increased $[14,19,21]$. Especially during the third trimester, aortocaval compression of the uterus will compromise the effectiveness of Cardiopulmonary Resuscitation (CPR) $[4,6,10,14,24,29]$. Although there is no robust evidence, effectiveness of CPR could be increased by tilting the mother $15-30^{\circ}$ or by manual displacement of the uterus, the latter being preferred $[1,4,6,7,14,17,19-21,23-26,29]$. If magnesium was given as a tocolytic prior to the arrest, it should be stopped and calcium should be administered instead. The focus of resuscitation

Table 2: Abbreviation of BEAUCHOPS.

\begin{tabular}{|c|}
\hline BEAUCHOPS \\
\hline Bleeding/DIC \\
\hline Embolism \\
\hline Anaesthetic complications \\
Uterine atony \\
\hline Cardiac disease \\
\hline Hypertension (pre-eclampsia and eclampsia) \\
\hline Others, namely 4H's and 4T's \\
\hline Placental abruption or Placenta previa \\
\hline Sepsis \\
\hline
\end{tabular}

should be towards identifying type and aetiology of arrest $[4,10,17,29]$. BEAUCHOPS (Table 2) is a helpful tool to assess possible causes of maternal cardiac arrest. Fetal anoxia mainly occurs after a traumatic hypovolemic maternal cardiac arrest, and is associated with a bad outcome $[1,11,23]$.

\section{Perimortem caesarean section (PCS)}

PCS consists of a caesarean section in a patient who is in extremis or is undergoing CPR [9]. Besides fetal survival, it can lead to a return of spontaneous circulation of the mother, making it a part of maternal resuscitation efforts $[4,10,14,29]$. Indeed, the best chance of fetal survival is maternal survival and that the latter can be achieved through fetal delivery [11]. However, in case of a traumatic cardiac arrest, PCS is more likely to save only the fetus $[6,30,31]$. Thus, PCS is recommended in a perimortem pregnant patient with potential fetal viability $[1,9,12,15-17,20,22,23,25,28,29]$, Besides PCS, thoracotomy with an open cardiac massage could also be performed. There is no consensus concerning the order in which PCS or thoracotomy should be performed.

\section{Conclusions}

Preventive education about the use of seatbelts and airbags together with screening for domestic abuse and depression are essential in the follow-up of pregnant patients $[5,6,10]$. In case of trauma, pregnant patients should always visit a physician since the degree of trauma is not predictive of the outcome $[4,10]$. There should be a communication concerning the benefit/risk of radiologic examinations. If no admission is needed, return precautions should be communicated such as vaginal bleeding, severe pain, ROM, decreased fetal movement and appropriate follow-up should be assured [3,17,21] .

\section{Declarations}

Ethics approval and consent to participate:

\section{Authors' contributions}

LD'A and MS designed the work. LD'A collected the data. LD'A and MS analysed the data. SV and MS substantively revised the manuscript. All authors read and approved the final manuscript.

\section{References}

1. Brown HL (2009) Trauma in pregnancy. Obstet Gynecol 114: 147-160. Link: https://bit.ly/2Q4Y7m2

2. Brown S, Mozurkewich E (2013) Trauma during pregnancy. Obstet Gyneco Clin North Am 40: 47-57. Link: https://bit.ly/2YdXATg

3. Cahill AG, Bastek JA, Stamilio DM, Odibo AO, Stevens E, et al. (2008) Minor trauma in pregnancy-is the evaluation unwarranted? Am J Obstet Gynecol 198: e1-e5. Link: https://bit.ly/317on5s

4. Hill CC (2009) Trauma in the obstetrical patient. Women's Health 5: 269-283. Link: https://bit.ly/3axb4P8

5. Petrone $P$, Jiménez-Morillas $P$, Axelrad A, Marini CP (2019) Traumatic injuries to the pregnant patient : a critical literature review. Eur J Trauma Emerg Surg 45: 383-392. Link: https://bit.ly/2Y7vLvW

6. Jain V, Chari R, Maslovitz S, Farine D, Bujold E, et al. (2015) Guidelines for the management of a pregnant trauma patient. J Obstet Gynaecol Canada 37: 553-574. Link: https://bit.ly/3iRoBnE

7. Wyant AR, Collett D (2013) Trauma in pregnancy: Diagnosis and management of two patients in one. JAAPA 26: 24-29.

8. Kuczkowski K (2008) A situation pregnant with danger: trauma in pregnancy SAJAA 14: 59-62. Link: https://bit.ly/321wRdU

9. Battaloglu E, Battaloglu E, Chu J, Porter K (2015) Obstetrics in trauma. Trauma 17: 17-23. Link: https://bit.ly/2YdUTBn

10. Hill CC, Pickinpaugh J (2008) Trauma and surgical emergencies in the obstetric patient. Surg Clin North Am 88: 421-440. Link: https://bit.ly/3axZenX

11. Raja AS, Zabbo CP (2012) Trauma in pregnancy. Emerg Med Clin North Am 30 937-948. Link: https://bit.ly/316qNS4

12. Mendez-Figueroa H, Dahlke JD, Vrees RA, Rouse DJ (2013) Trauma in pregnancy: An updated systematic review. Am J Obstet Gynecol 209: 1-10. Link: https://bit.ly/3kWRj8b

13. Chames MC, Pearlman MD (2008) Trauma during pregnancy: Outcomes and clinical management. Clin Obstet Gynecol 51: 398-408. Link: https://bit.ly/320ag1b

14. Rossignol M (2016) Trauma and pregnancy: What anesthesiologist should know. Anaesth Crit Care Pain Med 35: S27-S34. Link: https://bit.ly/34bbEkg

15. Oxford CM, Ludmir J (2009) Trauma in pregnancy. Clin Obstet Gynecol 52 611-629. Link: https://bit.ly/349aCFr

16. MacArthur B, Foley M, Gray K, Sisley A (2019) Trauma in pregnancy: A comprehensive approach to the mother and fetus. Am J Obstet Gynecol 220: 465-469. Link: https://bit.ly/2CANfJy

17. Sakamoto J, Michels C, Joshi N (2019) Trauma in pregnancy. Emerg Med Clin North Am 37: 317-338. Link: https://bit.ly/323xtzK

Citation: Argent LD, Verelst S, Sabbe M (2020) Management of the pregnant trauma patient: A literature study. Open J Trauma 4(1): 038-046. 
18. Einav S, Sela HY, Weiniger CF (2013) Management and outcomes of trauma during pregnancy. Anesthesiol Clin 31: 141-156. Link : https://bit.ly/3kV0h65

19. Petrone P, Marini CP (2015) Trauma in pregnant patients. Curr Probl Surg 52 : 330-351.

20. Pearce C, Martin SR (2016) Trauma and considerations unique to pregnancy Obstet Gynecol Clin North Am 43: 791-808. Link: https://bit.ly/349Fbe5

21. Murphy NJ, Quinlan JD (2014) Trauma in pregnancy: assessment management, and prevention. Am Fam Physician 90: 717-724. Link: https://bit.ly/32gSOG7

22. Lucia A, Dantoni SE (2016) Trauma management of the pregnant patient. Crit Care Clin 32: 109-117. Link: https://bit.ly/314wxeY

23. Warraich Q, Esen U (2009) Perimortem caesarean section. J Obstet Gynaeco 29: 690-693. Link: https://bit.ly/3g6IXHM

24. Benson MD, Padovano A, Bourjeily G, Zhou Y (2016) Maternal collapse Challenging the four-minute rule. EBioMedicine 6: 253-257. Link: https://bit.ly/31Vtwgc

25. Drukker L, Hants Y, Sharon E, Sela HY, Grisaru-Granovsky S (2014) Perimortem cesarean section for maternal and fetal salvage : concise review and protocol. Acta Obstet Gynecol Scand 93: 965-972. Link: https://bit.ly/3iKBvnc
26. Huls CK, Detlefs C (2018) Seminars in Perinatology Trauma in pregnancy. Semin Perinatol 42: 13-20. Link: https://bit.ly/3iO0K8c

27. Mirza FG, Devine PC, Gaddipati S (2010) Trauma in pregnancy : A systematic approach. Am J Perinatol 27: 579-586. Link: https://bit.ly/2YcBMYj

28. Barraco RD, Chiu WC, Clancy TV, Como JJ, Ebert JB, et al. (2010) Practice management guidelines for the diagnosis and management of injury in the pregnant patient: The EAST practice management guidelines work group. J Trauma 69: 211-214. Link: https://bit.ly/34goQ7L

29. Rose CH, Faksh A, Traynor KD, Cabrera D, Arendt KW, et al. (2015) Challenging the 4- to 5-minute rule : from perimortem cesarean to resuscitative hysterotomy. Am J Obstet Gynecol 213: 653-656. Link: https://bit.ly/2DTEmM1

30. Parry R, Asmussen T, Smith JE (2016) Perimortem caesarean section. Emerg Med J 33: 224-229. Link: https://bit.ly/323q6II

31. Moher D, Liberati A, Tetzlaff J, Altman DG (2009) Preferred Reporting Items fo Systematic Reviews and MetaAnalyses: The PRISMA Statement. PLoS Med 6: e1000097. Link: https://bit.ly/318d1yl
Discover a bigger Impact and Visibility of your article publication with Peertechz Publications

\section{Highlights}

* Signatory publisher of ORCID

- Signatory Publisher of DORA (San Francisco Declaration on Research Assessment)

* Articles archived in worlds' renowned service providers such as Portico, CNKI, AGRIS TDNet, Base (Bielefeld University Library), CrossRef, Scilit, J-Gate etc.

* Journals indexed in ICMJE, SHERPA/ROMEO, Google Scholar etc.

* OAI-PMH (Open Archives Initiative Protocol for Metadata Harvesting)

* Dedicated Editorial Board for every journal

* Accurate and rapid peer-review process

- Increased citations of published articles through promotions

- Reduced timeline for article publication

Submit your articles and experience a new surge in publication services (https://www.peertechz.com/submission).

Peertechz journals wishes everlasting success in your every endeavours.

Copyright: @ 2020 Argent LD, et al. This is an open-access article distributed under the terms of the Creative Commons Attribution License, which permits unrestricted use distribution, and reproduction in any medium, provided the original author and source are credited.

Citation: Argent LD, Verelst S, Sabbe M (2020) Management of the pregnant trauma patient: A literature study. Open J Trauma 4(1): 038-046. 